\title{
Percepciones de académicos sobre las dificultades para el fomento de la innovación y el emprendimiento: el caso de la FCFM de la Universidad de Chile
}

\author{
Natalia Zisis ${ }^{1}$, Patricio Moya ${ }^{2 *}$,Francisco Molina ${ }^{3}$
}

El objetivo de la presente investigación es describir y analizar la percepción de los profesores sobre las dificultades para fomentar la innovación y el emprendimiento en una institución de educación superior tradicional como lo es la Facultad de Ciencias Físicas y Matemáticas de la Universidad de Chile. El estudio, de carácter cualitativo, busca mejorar la toma de decisiones por parte de las instituciones involucradas. Se llevaron a cabo entrevistas semiestructuradas con los profesores de la Facultad. Los resultados señalan que mientras el término innovación no genera resistencia entre el profesorado, emprendimiento no produce el mismo consenso, ya que para algunos se relaciona con habilidades distintas a las que debieran ser formadas en la Universidad de Chile, dado su sello de alto prestigio y calidad. Esto a su vez implica que la exigencia y carga académica para los estudiantes sea muy alta, lo cual desincentiva la realización de proyectos extracurriculares de innovación y emprendimiento. Por otro lado, se reconoce un carácter extremadamente academicista, enfocado en el desarrollo de investigación no aplicada, altamente específica y desconectada de problemáticas del medio, que responde en gran medida a los incentivos establecidos para el quehacer académico. Esta cultura academicista se permea a los estudiantes, quienes encuentran pocas instancias de conexión con la realidad en su formación, de trabajo en equipo y de relación con otras disciplinas.

Palabras clave: innovación; emprendimiento; ingeniería; percepciones de los profesores.

\begin{abstract}
Title: Perceptions of academics on the difficulties for the promotion of innovation and entrepreneurship: The case of the FCFM of the University of Chile

The aim of this research is to describe and analyze the teachers' perception of the difficulties to foster innovation and entrepreneurship in a traditional higher education institution such as the Faculty of Physical and Mathematical Sciences of the University of Chile. The qualitative study seeks to provide information on this topic in order to improve decision making by the institutions involved. Semi-structured interviews were conducted with faculty teachers. The results indicate that while the term innovation does not generate resistance among teachers, the term entrepreneurship does not produce the same consensus, mainly because for some of the academics, it relates to skills that do not fit with Universidad de Chile standards, given its seal of prestige and quality. This also implies that the exigence and academic load for students is very high, which disincentives the development of extracurricular innovation and entrepreneurship projects. Additionally, the interviewees recognize a highly academic character of the institution, with focus on the development of non-applied, highly specific and detached from issues of the milieu research, which responds mainly to the structure of incentives that defines the academic job. This academic culture permeates to students, who find little instances of connection with reality, teamwork and relation with other disciplines within their formation.
\end{abstract}

Keywords: innovation; entrepreneurship; engineering; teachers' perceptions.

Submitted: July $14^{\text {th }} 2017 /$ Approved: November $30^{\text {rd }} 2017$

\section{Antecedentes generales}

En la actualidad, Chile se está enfrentando a desafíos que requieren de la participación de una serie de actores comprometidos con el progreso social y productivo. Dichos desafíos se encuentran relacionados, principalmente, con alcanzar los índices de calidad de vida que se encuentran en países desarrollados. Para lograrlo, se ha insistido en que debe existir una reorientación de las políticas públicas hacia la innovación y el emprendimiento, en la medida en que son las formas más efectivas en las que se puede contribuir con el progreso social y económico de los países (World Economic Forum, 2009). Durante los últimos años en Chile se ha incentivado, desde la esfera pública, el desarrollo en innovación y emprendimiento, puesto que se entiende que solo gracias a este tipo de iniciativas se pueden alcanzar niveles económicos y sociales propios de los países desarrollados.
La formación de profesionales en las áreas de ciencia y tecnología, y en particular en ingeniería, se ha transformado en uno de los elementos clave para el progreso de los países (Bjorklund y Colbeck, 2001). En la misma línea, la demanda por diversos servicios de ingeniería se encuentra en aumento y, además, la globalización de los mercados y los avances científico-tecnológicos han impactado la práctica ingenieril, ya que se integran ámbitos de actuación que antes no estaban considerados (Crawley et al., 2007). En este contexto, para adecuarse a estas demandas, la Corporación de Fomento a la Producción (Corfo) del Estado de Chile ha lanzado el programa "Nueva Ingeniería 2030", que tiene como uno de sus objetivos principales apoyar la formación de capacidades en innovación y emprendimiento en los alumnos de las carreras de ingeniería de las universidades que forman parte de este programa (como la Universidad de Chile o la Pontificia Universidad Católica de Chile).

(1) Investigadora Independiente

(2) Laboratorio de Innovación y Emprendimiento - Facultad de Ciencias Físicas y Matemáticas - Universidad de Chile

(3) Facultad de Ciencias Físicas y Matemáticas -Universidad de Chile

*Autor de correspondencia: pmoyam@uchile.cl 
La Universidad de Chile es la más antigua y, a la vez, una de las principales instituciones de educación superior en el país. Por su parte, la Facultad de Ciencias Físicas y Matemáticas (FCFM), donde se dictan las carreras de ingeniería civil en la Universidad, cuenta con alrededor de 4.900 estudiantes de grado, 1.200 de posgrado y 330 académicos (220 profesores a jornada completa, de los cuales el $97 \%$ posee el grado de doctor). En cuanto a la capacidad científica que se produce en el país, la Universidad produce el 25\% del total, mientras que la FCFM produce el 6\%. Sin embargo, a pesar de estas características, la Universidad reconoce que tanto la transferencia tecnológica como la innovación y el emprendimiento basado en ciencia y tecnología son las áreas más débiles del desarrollo institucional. Además, como la propia Facultad reconoce, la formación del alumnado en los conceptos de innovación y emprendimiento no ha sido una de las preocupaciones de la institución, y solo por medio de la ejecución del programa "Nueva Ingeniería para el 2030", se han comenzado a integrar dichas competencias en los planes formativos. La perspectiva adoptada para llevar a cabo este cometido se relaciona con el emprendimiento dirigido por la innovación (innovation-driven entrepreneurship) que se caracteriza por perseguir oportunidades orientadas a llevar a los clientes nuevas innovaciones que tienen claras ventajas competitivas y un gran potencial de crecimiento. En este contexto, la innovación es entendida como el poder insertar nuevas ideas en el mercado, la técnica o el modelo de negocios (Aulet y Murray, 2013). Como sea, dados los rasgos de la FCFM, la innovación y el emprendimiento no pueden ser entendidos si no integran un fuerte componente de ciencia y tecnología.

Considerando esta nueva empresa en la que se embarca la institución, es interesante preguntarse por los elementos que podrían facilitar o dificultar su éxito. Según la literatura, uno de los factores más críticos para explicar el éxito de la introducción de la innovación y emprendimiento en las universidades es la percepción de académicos y estudiantes hacia el emprendimiento (Guerrero y Urbano, 2010). Sin embargo, hasta ahora los estudios en el área se han centrado en describir las percepciones de los estudiantes sobre la innovación y emprendimiento en general (Souitaris, Zerbatini y Al-Laham, 2007; Davey, Plewa y Struwig, 2011) y no se ha considerado de igual manera la imagen que los académicos podrían tener al respecto. Por tal razón, el objetivo principal de nuestra investigación es describir y analizar la percepción de los profesores sobre las dificultades para fomentar la innovación y el emprendimiento en una institución de educación superior tradicional como lo es la FCFM de la Universidad de Chile. El estudio, de carácter cualitativo, busca entregar información sobre este tema con el fin de mejorar la toma de decisiones por parte de las instituciones involucradas.

\section{Marco teórico}

Los obstáculos para fomentar la innovación y el emprendimiento en las universidades

Son diferentes las dificultades que enfrentan las universidades, sobre todo tradicionales, en pos del fomento de la innovación y el emprendimiento. En primer lugar, destaca la subordinación que presentan con respecto al Estado, en la medida en que son fuertemente dependientes de este, tanto regulatoria como financieramente (Mora y Villareal, 2001). Esto implica, en términos financieros, una falta de incentivo por mudar hacia un sistema que busque vías alternativas de financiamiento y al mismo tiempo responda a las demandas de la sociedad y del mercado mediante el emprendimiento. Al mismo tiempo, según Mora y Villarreal (2001), esta dependencia se traduce en una lentitud burocrática para llevar a cabo cambios innovadores, así como en poca libertad para tomar decisiones respecto al personal. Se percibe, así, un desbalance cada vez mayor entre lo que se espera y demanda (enseñanza e investigación) de las universidades y su capacidad efectiva de responder, debido a la poca diversificación que tienen respecto al origen de su financiamiento y a la mantención de estructuras internas rígidas que son herencia de un pasado más simple para la educación superior de elite (Clark, 2001). En segundo lugar, los procesos de toma de decisión en las universidades tradicionales tienden a ser lentos e inefectivos dada la estructura de colegialidad que lo determina. El poder de decisión se reparte entre múltiples entidades separadas (facultades, escuelas o departamentos) que tienen privilegios individuales y que apenas se relacionan entre sí. Finalmente, esto se traduce en que los recursos se dirigen a la mantención más que a inducir y apoyar algún cambio (Oleksiyenko, 2002), y a que la responsabilidad directa de las decisiones operacionales no esté bien definida (Mora y Villareal, 2001).

Por último, influye el carácter mismo de las universidades tradicionales y su pertenencia al sector público. La existencia de emprendimientos en el sector público es ya percibida como una paradoja, ya que para muchos el sector público se percibe como sinónimo de burocratización y se asocia a comportamientos rutinarios, evitación del riesgo y falta de iniciativa (Bernier y Hafsi, 2007). A esto se suma la dificultad de medir ganancias, el establecimiento de objetivos poco definidos y complejos, los problemas de la acción colectiva y la coerción que se da en instituciones públicas (Klein y Zur 2009). A su vez, Morris, Coombes, Schindehutte y Allen (2007), refiriéndose al mismo tipo de instituciones, agregan que los espacios de trabajo en los cuales los errores son tratados como oportunidades de aprendizaje, se alienta la iniciativa individual, se pone énfasis en la excelencia, los trabajadores se esfuerzan por el bien del equipo y existe una búsqueda continua por nuevas maneras de mejorar las operaciones, tenderán a tener niveles de emprendimiento más altos.

\section{La universidad emprendedora}

En la actualidad, se ha asumido que la universidad, junto con la enseñanza y la investigación, se encuentra cumpliendo una tercera misión: contribuir a la sociedad y al desarrollo económico de manera más directa. Las líneas de investigación en el área sugieren que si bien las universidades comparten objetivos y metas, tienen diferentes historias, tradiciones y estructuras organizacionales que moldean las formas en las que se lleva a cabo dicha tercera misión (Martinelli, Meyer y von Tunzelmann, 2008).

La universidad emprendedora (entrepreneurial university) juega un rol fundamental tanto como institución productora de información 
como diseminadora de la misma. En esta línea, se trata de universidades que han mejorado diferentes mecanismos tanto para contribuir al desarrollo regional como para incrementar sus ingresos (Guerrero y Urbano, 2010). Además, la universidad no se concibe solo como una promotora de una serie de medidas de apoyo al emprendimiento, sino que también es una desarrolladora de una serie de técnicas administrativas y estrategias competitivas. Así, estas universidades están involucradas en alianzas, redes de contacto y otros tipos de relaciones con organizaciones públicas y privadas. Estas relaciones se transforman en un verdadero paraguas para la interacción y colaboración que puede existir entre los diferentes elementos de un sistema nacional de emprendimiento. Los estudios identifican dos tipos de factores que confluyen en el desempeño de una universidad emprendedora: del medio e internos. Por un lado, entre los factores del medio se cuentan los factores formales y los informales. Mientras los primeros se relacionan con, por ejemplo, la organización emprendedora, las medidas de apoyo para el emprendimiento y la educación en emprendimiento; los segundos implican las actitudes de la comunidad hacia el emprendimiento, las metodologías para su enseñanza y, por último, los sistemas de incentivo. Por otro lado, los factores internos se dividen entre recursos (humanos, financieros, físicos y comerciales) y capacidades (estatus, redes de contacto y localización) (Guerrero y Urbano, 2010). El modelo más citado de este tipo de universidad es el que propone Clark (1998, cit. en Brunner, 2006). Más que responder a una propuesta normativa, destinada a incrementar el managerialismo de su gestión, Clark caracteriza un tipo de universidad que durante la década de los '90 habría enfrentado exitosamente los procesos de adaptación al nuevo entorno, en el cual la entrada en vigencia del nuevo modelo neoliberal obligó a las economías de casi todo el mundo a abrir sus mercados a la competencia externa y a reducir la intervención del Estado en ellas (Clark, 2001). Esta adaptación por parte de las universidades se realizó mediante la adopción de cambios que les permitieron elevar sus capacidades de respuesta y acelerar su trayectoria adaptativa (Brunner, 2006).

Sin embargo, existe una fuerte resistencia cultural frente a este tema. El factor más crítico para explicar el desarrollo de universidades emprendedoras es la percepción de académicos y estudiantes hacia el emprendimiento (Guerrero y Urbano, 2010). Cada comunidad universitaria es única y sus actitudes hacia el emprendimiento se definen por una combinación de factores, tales como educación en emprendimiento, metodologías de enseñanza, modelos a seguir y sistemas de incentivos (Guerrero y Urbano, 2010). El legado de las universidades tradicionales es que aún hoy muchas universidades no ven el emprendimiento como una oportunidad, sino más bien como una amenaza. Esto porque su sistema de incentivos tiene más que ver con el estatus y el prestigio (lo cual se traduce en financiamiento público), que con emprendimientos que conlleven financiamiento por otras vías (Oleksiyenko, 2002). Además, en las universidades tradicionales aún se busca realizar investigación teórica, que responden a motivaciones intrínsecas de satisfacción de los académicos, para satisfacer su curiosidad en el tema, y pocas veces motivada por incentivos fuera de la investigación misma (McInnis, 2001). La cultura de las universidades tradicionales tiende a ser altamente respetuosa de la autonomía indi- vidual, poco amenazante y segura para los académicos. En general los académicos tienen que dar poca cuenta de su labor a sus pares y superiores, por lo cual cuando se les exige ser más flexibles, adaptables, rápidos o innovadores para lograr la nueva misión de transformar a la universidad en una emprendedora, perciben el emprendimiento como un término negativo (Oleksiyenko, 2002). El fenómeno de las universidades emprendedoras ha emergido con una estrategia común que se enfoca en ser emprendedor en todo nivel implicado en la universidad. Sin embargo, las universidades son organizaciones complejas que comprenden un gran número de comunidades de práctica sobrepuestas y anidadas y el beneficio económico para el área local es poco visible.

\section{Método}

Considerando que nuestro objeto de estudio son las percepciones de profesores de una institución tradicional respecto a un fenómeno complejo y que implica construcciones de significado por parte de una comunidad, fue necesario adoptar un enfoque que permitiera observar el fenómeno de manera holística, sin reducir a los actores involucrados, escenarios, contextos, etc., a variables (Taylor y Bogdan, 2010). Por esto, el tipo de investigación adoptado fue cualitativo, ya que se busca determinar cómo se crea la experiencia social y cómo se construyen significados comunes; a diferencia de la investigación cuantitativa, la cual busca la cuantificación de ciertos fenómenos en cuanto a su frecuencia, cantidad, intensidad, etc. (Denzin y Lincoln, 2000). La metodología escogida es, además, particularmente útil cuando se conoce poco sobre el objeto de estudio y cuando se desea acceder a procesos subjetivos y la perspectiva propia de los actores sociales, como es el caso de nuestro estudio (Krause, 1995).

\section{Muestra}

Dada la especificidad de la temática a abordar en este estudio (desarrollo de proyectos por parte de los estudiantes), la muestra fue seleccionada según los principios de pertinencia y adecuación (Flick, 2002), es decir, acorde al aporte que los casos puedan significar en términos del tipo de información que estos representan para el objetivo de la investigación. Por esta razón, se seleccionaron académicos que involucraran estudiantes en sus proyectos, realizaran proyectos en sus cursos o fueran reconocidos por los estudiantes como puntos de contacto a quienes dirigirse para poder realizar sus propios proyectos. Se entiende, por tanto, que el desarrollo de proyectos se relaciona, de alguna manera, con los procesos intrínsecos asociados a la innovación y al emprendimiento (Brady y Hobday, 2011).

Dado que la muestra fue intencionada para cumplir con el objetivo de la investigación, no es posible afirmar que los resultados de este estudio son representativos de la opinión de todos los académicos de la FCFM. Sin embargo, el acercamiento de los académicos entrevistados a la realización de proyectos o participación en ellos por parte de los estudiantes permite una mayor profundización en este fenómeno. En vista de lo acotado del universo de estudio y de que se buscaba esta característica particular en la muestra, el muestreo se llevó a cabo a partir de informantes clave en un principio y luego mediante 'bola 
de nieve', es decir, a partir de referencias de los mismos entrevistados (Noy, 2008). Considerando lo anterior, se logró generar una muestra compuesta por 21 académicos, heterogénea en términos de su departamento de procedencia y del tipo de jornada laboral que cumplen en la facultad.

\section{Levantamiento de información}

Se llevaron a cabo entrevistas semiestructuradas en la medida en que estas presentan una gran capacidad para lograr una riqueza informativa contextualizada y holística (Flick, 2002). Este instrumento de recolección de información se utiliza cuando se asume que el entrevistado tiene un caudal complejo de conocimientos sobre el asunto, y que este conocimiento incluye supuestos que son explícitos e inmediatos, que son expresados espontáneamente al responder a una pregunta abierta, pero que también pueden ser complementados por supuestos implícitos que son más invisibles y en los cuales se debe indagar con preguntas que no necesariamente son planteadas a priori (Flick, 2002). Puesto que el fenómeno que se busca observar en este estudio no sólo no ha sido estudiado previamente en este contexto, sino que ha sido poco problematizado, es de esperar que existan varios supuestos implícitos que no han sido incorporados al discurso por parte de los entrevistados, y que pueden surgir a partir de una entrevista semiestructurada. Se utilizó, por tanto, una pauta de entrevista para su aplicación, la cual en primera instancia fue desarrollada considerando el objetivo de la investigación.

\section{Análisis de resultados}

Para el análisis de resultados se realizó una codificación abierta, según la cual se trata de expresar los datos y fenómenos en forma de conceptos, asignando anotaciones (códigos) a cada idea expresada por los entrevistados y posteriormente analizando el conjunto de expresiones como un concepto (Flick, 2002).

\section{Resultados}

Los resultados se presentarán de acuerdo a las categorías más importantes que nos permiten organizar de mejor manera las percepciones de los profesores con respecto a diversos factores que determinan la promoción de la innovación y el emprendimiento.

\section{Innovación}

Respecto al término innovación, no se genera resistencia alguna: todos lo perciben como algo positivo y necesario de implementar en la formación de los estudiantes de la facultad. En general, relacionan la innovación con hacer algo nuevo, lo cual puede referirse tanto a plantear algo distinto a lo que se encuentra en la industria como a hacer lo mismo con menos recursos. Asimismo, para la mayoría de los académicos, la innovación se relaciona directamente con el desarrollo de ciencia y tecnología, a hacer uso de las capacidades técnicas instaladas en la facultad para generar nuevas soluciones. La idea de que la innovación que se engendra en la facultad no puede ser cualquier tipo de innovación reafirma el carácter tradicional de la institución. Lo anterior se puede observar en la siguiente cita:
"Si aquí hay gente con una cantidad de privilegios impresionantes en la sociedad, con una fortuna impresionante respecto a las capacidades que pueden tener, no puede ser que lo que salen a hacer al mundo sea a vender ropa." (Profesor 9)

Además, la innovación se observa, para algunos, como parte de un círculo virtuoso que debe generarse entre la industria y la universidad. Se percibe que la industria chilena es incapaz de generar soluciones innovadoras, y para que éstas se generen deben gestarse en el ámbito de la academia, es decir, se vislumbra como parte de la misión de la universidad generar investigación aplicada e innovadora que permita la solución de problemáticas reales.

Sin embargo, también se manifiesta que la facultad no ha logrado aún estar a la altura de esta tarea, ya que son muy pocos los académicos que ven un valor en generar investigación que pueda ser aplicada. Esta opinión se observa claramente en la siguiente cita:

"Nunca ha habido en el departamento, esto de, hacer un camino alternativo, que sea de alguien que se dedique a investigar algo porque le interesa sacar una aplicación de eso y llevarlo a la industria, por ejemplo, a una aplicación industrial, a la creación de una empresa, o a la creación de un servicio, de un producto, etc., que lo lleve al mercado, o que se lleve a la implementación en el ámbito que sea." (Profesor 19)

\section{Emprendimiento}

El término 'emprendimiento' genera más polémica entre los entrevistados. Mientras hay quienes lo ven como algo completamente positivo y que se debe fomentar (a pesar de que reconocen que es difícil de implementarlo en la cultura institucional actual), hay quienes lo observan con cierto dejo de desconfianza.

Dadas las características de la muestra, la mayoría de los entrevistados percibe el emprendimiento como algo positivo, pero poco desarrollado en la facultad. Se manifiesta una sensación de, en palabras de un entrevistado, estar quedándonos atrás en este tema, respecto a otras universidades. Sin embargo, también se vislumbra una sensación de optimismo, lo que se relaciona en gran medida con el desarrollo del Proyecto Ingeniería 2030. Se empiezan a distinguir ciertas iniciativas en la facultad que van en la línea de fomentar el emprendimiento, así como un mayor interés por parte de los estudiantes por estas temáticas. Asimismo, muchos entrevistados manifiestan que el fomento del emprendimiento viene como iniciativa de las nuevas generaciones de profesores que van llegando, por lo que se aprecia una tendencia hacia esa dirección. En este sentido, se hace el reparo de que estas iniciativas se vislumbran sólo a nivel de facultad, mientras que en el resto de la universidad todavía no se percibe esta tendencia. Sin embargo, en la misma facultad tampoco se advierte el sentido de urgencia que, para quienes la perciben de manera positiva, debería tener esta temática, lo cual se puede rescatar de la siguiente cita:

"Mi visión particular es que hay una percepción o una intuición de que son tópicos relevantes, para algunos, otros no tanto, pero yo lo que sí creo en común es que no hay una urgencia, nadie cree que es algo urgente que tengamos que resolver o atacar." (Profesor 9) 
Además, los académicos perciben que los estudiantes vislumbran pocas oportunidades para emprender en la facultad, y que se han intentado instalar ciertas estructuras que lo hubieran permitido en el pasado, pero que han fallado. Esto ha llevado a una sensación general de que el emprendimiento no es para nosotros y sí para otras universidades, que no cargan con el peso de la dependencia que existe con el Estado, como es el caso de las universidades privadas.

En general, al igual que en el caso del concepto de innovación, el emprendimiento se tiende a relacionar, en el contexto de la facultad, con el desarrollo de ciencia y tecnología. Asimismo, se le da gran importancia al potencial de resolver problemáticas reales que puedan tener los emprendimientos, aunque no se identifique completamente emprendimiento con innovación, sino más bien con la implementación y operación de alguna iniciativa.

Incluso quienes tienen una visión positiva del emprendimiento, manifiestan ciertos reparos al respecto. Por un lado muchos reconocen que no todos los estudiantes pueden ser emprendedores y que hay cierto riesgo en inculcarles la necesidad de emprender, ya que la posibilidad de fracaso es tremendamente elevada. Además, observan que es especialmente difícil ser emprendedor en Chile, en la medida en que se percibe una cultura muy conservadora en este sentido: el mercado no espera emprendedores, sino empleados y la innovación y el emprendimiento en general son difíciles de instaurar de manera sustentable en una industria como la chilena, que carece de un ecosistema robusto de innovación y emprendimiento. Un ejemplo de esto es el hecho de que gran parte de la innovación y el emprendimiento son financiados con fondos públicos. Esta visión negativa del ecosistema de emprendimiento en Chile se observa en la siguiente cita:

“(...) hay que asumir que nuestro mercado laboral, no lo promueve mucho [el emprendimiento], la verdad. Nuestro mercado laboral, las empresas, son súper conservadoras, si tú llegas con mucha innovación, te quedan mirando raro y te dicen, 'oye, ya pero, y eso dónde funciona', 'quién lo ha probado', 'quién le saca los errores' (...) Y eso justamente va en contra de la innovación, porque en el fondo te están obligando a usar lo conocido, lo aprobado, lo que tiene soporte, y con el emprendimiento yo creo que es peor." (Profesor 3)

Además, en general se percibe una cultura institucional que presenta una gran resistencia a este nuevo término, por variadas razones. Hay quienes observan que de manera generalizada, en la facultad no se habla de emprendimiento, no es tema, puesto que para los académicos escapa a su quehacer y no le dan mayor importancia. En este sentido, se observa un interés mucho mayor por parte de los estudiantes por el tema que de parte de los académicos, quienes en su mayoría no se hacen cargo del interés de los estudiantes por estas temáticas. Los entrevistados señalan acerca de los académicos una mentalidad muy conservadora, cientificista y, valga la redundancia, académica, y que para la mayoría de ellos el emprendimiento no es algo que se debiera fomentar, porque ocurre de manera espontánea. Esta idea se ve reflejada en la siguiente cita:
"P: Yo creo que los estudiantes están motivados y yo creo que para los profesores es un cacho [problema] más. Y lo digo bien responsablemente. Yo soy uno de los profesores que está metido en temas de tratar de empujar.

\section{E: ¿Un cacho [problema] en el sentido de responsabilidad?}

P: No, un cacho [problema]. Algo que no corresponde a lo que uno hace. Porque en el fondo aqui se han contratado investigadores, no se han contratado gente emprendedora, no se han contratado gente innovadora en el sentido de transferencia tecnológica (...)" (Profesor 15)

Por otro lado, los entrevistados identifican también que hay opiniones que más allá de mantenerse neutrales frente al emprendimiento, o no querer involucrarse en estas temáticas, se oponen a él. Hay quienes se oponen porque identifican emprendimiento con lucro, y consideran que esto no se alinea con la misión de la universidad y con el quehacer académico. Para otros, un enfoque en el emprendimiento implicaría además una baja en la calidad técnica de la facultad, ya que se exacerban ciertas habilidades que no son las relevantes de desarrollar en un ingeniero, en desmedro de la exigencia técnica. Esta idea se expone en la siguiente cita:

"Los profes más clásicos lo que sentían es que esta cuestión [Proyecto Ingeniería 2030] finalmente estaba casi que hecha para que se bajara la vara, se bajara la exigencia de los alumnos, porque hay que integrar cosas que tienen que ver con habilidades profesionales, habilidades blandas, que les llamaban antes, cachai' [sabes]. (...) Yo creo que siempre se ha mirado con suspicacia, siempre, siempre, como que 'soi' [eres] medio chanta [charlatán], o estai' [estás] sacando la vuelta', y yo creo que va a costar harto [mucho]." (Profesor 4)

Se observa entonces que en gran medida el resquemor de ciertos académicos hacia el tema del emprendimiento se deriva de relacionarlo con habilidades que consideran que no son las que un ingeniero debiera desarrollar, y menos aún, uno de la Universidad de Chile. El interés por el lucro, la habilidad de vender la pomada [engañar], tener labia, se perciben negativamente y se relacionan directamente con esta temática para los académicos más 'conservadores'. Sin embargo, se observa al mismo tiempo una tendencia al cambio en estas corrientes de opinión:

"Yo creo que en el tema del emprendimiento había más resistencia. Una especie como de discurso, había sospecha de que esto fuera como una especie de... De que debilitara la pureza ingenieril, o como que la hiciera más blanda, o que los intereses comerciales se pusieran sobre los intereses de la innovación, o desarrollo puro, entonces eso yo creo que ha ido cambiando. Yo creo que estamos en posición de que eso está cambiando. Hay gente que tiene más apertura." (Profesor 10) 


\section{Académicos}

Como se explicaba en la sección anterior, los entrevistados advierten que los académicos de la facultad en general están profundamente desconectados de la innovación y, especialmente, emprendimiento. Esto lo explican a partir del carácter de la academia en general y los incentivos que se imponen en esta esfera: para mantenerse y ascender en este ámbito tan competitivo, la principal tarea del académico es publicar artículos de investigación en revistas de corriente principal de manera regular. Los académicos en general ocupan su tiempo, además de preparar y hacer clases, en conseguir financiamiento para llevar a cabo sus proyectos de investigación, los cuales pocas veces tienen pretensiones de aplicarse a algún emprendimiento o algo que salga de la esfera académica.

“(... si a ti te evalúan por las clases que haces o por los papers que publicas, tampoco te puedes dedicar a eso [apoyar proyectos estudiantiles], así es que yo creo que la única manera en que sea sustentable OpenLab, es que vaya de la mano con un cambio, por un lado en la manera en que se evalúa a los profesores, y también a los estudiantes." (Profesor 8)

Además, se percibe que muy pocos académicos, a excepción de los part-time, mantienen alguna relación con la industria, con problemáticas reales, y en general, tienen poca experiencia profesional. Se dedican a temáticas de enorme especificidad, y están tan desconectados con el mundo laboral que no vislumbran las oportunidades de aplicación de la investigación que realizan. Esta idea se expone en la siguiente cita, de parte de un profesor part-time que se desempeñó mucho tiempo fuera de la universidad:

"Muy pocos profes acá tienen experiencia profesional. Entonces claro, en el mundo de la investigación es súper relevante que tú seas hiper experto en algo súper complejo. Porque eso es lo que te permite destacar. Publicar en un tema que nadie más pública, porque en el mundo profesional los problemas no son teóricamente tan complejos. Si no que, lo difícil es cómo esa cosa técnica se compatibiliza con los recursos que tienes, o con las restricciones de mercado, que tienes una comunidad que no quiere el proyecto. Es la conjunción de cosas la que es compleja. Entonces yo creo que a nuestros ingenieros les hace falta una visión más amplia. O sea, está bien, tú eres ingeniero mecánico, y tienes que resolver los problemas mecánicos, pero el proyecto es mucho más que eso." (Profesor 3)

En consonancia con lo anterior, el nivel de especificidad de la investigación realizada por los académicos desincentiva también que los académicos se relacionen entre sí y realicen proyectos en conjunto. Este fenómeno se da no sólo a nivel de facultad, sino que incluso a nivel de departamentos: se observa muy poco trabajo en equipo o interdisciplinario, ya que las estructuras que modelan la academia lo desalientan.

Considerando lo anterior, los entrevistados indican que difícilmente los académicos de la facultad se encuentran en una posición como para fomentar la innovación y el emprendimiento, en vista de lo alejados que están ellos mismos de los elementos que permiten su desarrollo, como son el trabajo interdisciplinario y la conexión con problemáticas reales.
Carga académica y nivel de exigencia

La mayoría de los entrevistados se refieren a la carga académica de los estudiantes como uno de los principales obstáculos para que éstos desarrollen proyectos extracurriculares. Consideran que los estudiantes que pueden llevar a cabo proyectos fuera del marco curricular son excepciones, dada la alta demanda en tiempo que implican las actividades curriculares. Además, quienes decidan llevar a cabo estos proyectos se relacionarían con dos tipos de estudiantes: aquellos que están dispuestos a bajar su rendimiento académico o aquellos que son considerados como 'brillantes'. Algunos de los entrevistados consideran que en la FCFM, más que incentivarse que los estudiantes realicen actividades formativas (en un sentido amplio) fuera de lo curricular, esto es castigado, como se observa en la siguiente cita:

"Así como las facilidades para que los alumnos hagan cosas fuera de Beauchef, en el fondo que están no sé, representando a la universidad en un concurso o haciendo un proyecto externo, a nivel internacional incluso, en vez de ayudarlos se les castiga, cachai'[sabes]. Es como 'no, fregaste [no tienes posibilidad], ninguna posibilidad de que el control lo tomes en otra fecha, tienes que dar el examen igual que todos los demás que flojearon [fueron perezosos], o que dieron un certificado falso"'. (Profesor 4)

La contracara de esta situación es que muchos académicos estiman que es difícil realizar proyectos con estudiantes de grado, dada la poca disponibilidad de tiempo que tienen. Es especialmente difícil si estos proyectos tienen contrapartes que fijan ciertos compromisos en su desarrollo:

"Hay un riesgo no menor de contar con estudiantes para elaborar proyectos donde hay contratos de por medio, compromisos con terceros, con gente externa, grandes empresas, etc. En términos, por ejemplo, de cumplimiento de plazos o de control de calidad es muy complejo, o sea, porque el estudiante no tiene muchas veces el tiempo para poder realizarlo." (Profesor 18)

Por otro lado, se identifica una nueva dificultad. Muchos estudiantes que están entusiasmados con la realización de algún proyecto extracurricular o que quieren dar continuidad a algún proyecto que comenzaron en el marco de algún curso, terminan por abandonarlo porque deben atender a sus labores académicas. Una solución que algunos académicos ven a esta situación es integrar la realización de proyectos en el marco curricular o reconocer la realización de estos proyectos académicamente, créditos académicos, por ejemplo.Lo anterior se refleja en la siguiente cita:

"Al no ser parte de un curso, y al no estar siendo evaluados, la prioridad y la intención de emprender de ellos baja mucho, porque al principio parten el semestre, que pasa todos los semestres... 'no, queremos continuar el proyecto', 'oye, ¿nos pueden ayudar?', nosotros 'sí, obvio', pero llegan a la semana cinco y están reventados con Investigación de Operaciones, con Marketing, con Finanzas, con miles de otros ramos y al final como ya no es parte de un curso, ellos tienen que hacer la elección, o cumplo con mis deberes como estudiante, para tratar de salvar mis ramos, me dedico a emprender. Entonces creo que ahí hay una incompatibilidad, entre cómo está diseñada la vida de un estudiante, normal, con el impulso para emprender." (Profesor 2) 
Para muchos de los entrevistados, la alta calidad técnica y científica que caracteriza la formación de la facultad tiene un lado negativo: un nivel de exigencia que impide que los estudiantes lleven a cabo proyectos extracurriculares. Algunos consideran que este nivel de exigencia se anida en (y potencia) una cultura de desconfianza entre los profesores y los estudiantes. Es decir, el profesor considera que fuera del marco curricular lo que el estudiante hace es flojear [holgazanear], por lo que consume todo su tiempo con actividades curriculares, y por su parte el estudiante responde a esto cumpliendo con el mínimo, conformándose con "pasar los ramos". Esta cultura vuelve estériles los esfuerzos de innovación y emprendimiento. Para muchos de los entrevistados, en lugar de fomentarse estas iniciativas, se las desincentiva, lo que se observa en el cambio de actitud que tienen los estudiantes a lo largo de su carrera, como se enseña en la siguiente cita:

"Mi tesis es que los alumnos al tercer año son muchísimo menos emprendedores e innovadores que los alumnos de primer año. Esa es mi tesis, yo creo que lo que hacemos en vez de ayudarlos, como que los achatamos." (Profesor 4)

En este sentido, el nivel de exigencia es tal que quienes quieran llevar a cabo proyectos extracurriculares o bien tienen que ser "brillantes", o correr el riesgo de reprobar ramos o bajar sus notas:

“(...) lo que se tiene que fortalecer es la estructura académica para que eso [innovación y emprendimiento] sea reconocido, y para que no sea un extra, porque si no quizás lo van a poder hacer los estudiantes que son ultra ultra brillantes nomás po', que pueden tomar tres cursos o cuatros cursos normales, hacer las tareas, los controles, los exámenes, etc... Es como perder el recurso, si les vas a exigir el doble a la gente porque haga innovación, no tiene mucho sentido." (Profesor 8)

Para algunos, el nivel de exigencia es tal que no sólo es innecesario para la formación de los ingenieros, sino que incluso ha derivado en problemas psicológicos entre el estudiantado:

"El nivel de exigencia, y todo esto se sabe, pero nadie hace nada. O sea tienes una unidad de calidad de vida que tiene tres psicólogos, para seis mil alumnos, es imposible. Y por qué te digo esto, para fundar que el nivel de exigencia es tal, de que hay personas que se matan por el fracaso que va a ser, fracasar en la universidad. Es heavy. Entonces yo creo que eso tiene mucha relación, con la no cultura de emprendimiento que hay acá. Porque en el fondo cómo emprendes si no me dejan respirar (...)" (Profesor 2)

\section{Conclusiones}

Una universidad pública, tradicional y de excelencia

Para los entrevistados, el hecho de que la facultad pertenezca a la universidad más tradicional y antigua de Chile, considerada de excelencia en los ránkings nacionales, tiene varias implicancias en relación con la posibilidad de fomentar la innovación y el emprendimiento en la institución. En primer lugar, cabe destacar el "rol público" que se le adjudica a la universidad. La universidad tiene la responsabilidad social de ser un motor de desarrollo y abordar las problemáticas sociales que aquejan al país. Esto, por un lado, implica que se debiesen llevar a cabo iniciativas en la facultad que tuvieran algún impacto en el medio, lo cual, según los entrevistados, hoy no sucede en gran medida. La desconexión con la realidad por parte tanto de estudiantes como académicos es un punto de común acuerdo entre los profesores entrevistados. Por otro lado, este "rol público" implica también que lo que se espera de la Universidad de Chile es distinto a lo que se espera y acepta de otras universidades del país, en el sentido de que, por ejemplo, llevar a cabo proyectos que más que el bien común apunten al lucro y enriquecimiento, a los cuales muchas veces se asocia el término 'emprendimiento', no corresponden a esta institución, aunque sí puedan corresponder a otras.

En segundo lugar, la Universidad de Chile se representa en la cultura institucional como altamente prestigiosa y de excelencia. Esto, según los entrevistados, tiene una implicancia que no es favorable al fomento de la innovación y el emprendimiento y que se relaciona con la carga académica que se imprime sobre los estudiantes.

Por último, como preveen Mora y Villareal (2001), ser una institución tradicional y de dependencia estatal conlleva una burocratización de todos los procesos internos lo que es contraproducente para el desarrollo de la innovación y el emprendimiento. Los entrevistados reconocen que los tiempos burocráticos de la institución no se condicen con la rapidez que deben tener los procesos de asociados a la innovación y el emprendimiento, especialmente si se busca impactar al mercado. Sin embargo, no necesariamente relacionan explícitamente este problema con el hecho de que la universidad sea pública, sino que simplemente es una de las características que se le adjudican a la institución.

\section{Carácter academicista de la facultad}

Los entrevistados perciben que impera una cultura altamente academicista en la facultad, lo cual podría explicarse por tres factores. En primer lugar, como señalan Guerrero y Urbano (2010), el sistema de incentivos de una universidad es un factor que determina las actitudes hacia el emprendimiento de una comunidad universitaria. En este caso, los incentivos que tienen los académicos apuntan a que éstos se dediquen completamente a la docencia y a la investigación, en la mayoría de los casos, teórica. Se espera de ellos que destaquen en su área de expertise como investigadores, generando un número de artículos publicados en revistas indexadas, lo que generalmente implica llevar la investigación al máximo grado de especificidad en lugar de cooperar con otros (ya que tampoco tienen incentivos para la cooperación) en buscar aplicaciones prácticas para estos avances científico tecnológicos. Como señala McInnis (2001), las universidades tradicionales favorecen la autonomía individual de los académicos y se identifica con un espacio poco amenazante y seguro para ellos, en el cual pueden realizar investigación teórica que responda a sus motivaciones intrínsecas y pocas veces motivada por incentivos fuera de la investigación misma.

En segundo lugar, la exacerbación del academicismo en la facultad responde también a la manera en que se representa el rol del académico, en cuanto a lo que le corresponde y no hacer. En este sentido, fomentar la innovación y, en especial, el emprendimiento, escapa a 
las tareas que se esperan del académico, quien debiera dedicarse en un $100 \%$ a la docencia e investigación. Como lo expresa uno de los entrevistados, pastelero a tus pasteles: no se puede esperar de los académicos que se hagan cargo de esta nueva empresa que se ha decidido desarrollar en la facultad, ya que en primera instancia no fueron contratados para eso. Como explica Oleksiyenko (2002), exigirles esto podría incluso hacerles percibir el tema del emprendimiento como una amenaza a su forma tradicional de trabajar más que algo que se deba fomentar en la facultad. Asimismo, hay aspectos legales que desmotivarían el desarrollo emprendedor por parte de los académicos en las universidades estatales:

Incluso hay elementos en la propia ley chilena como el Estatuto Administrativo o la Ley de Probidad, que regula el régimen del Funcionario Público, y que podrían impedir que los académicos de universidades estatales realicen actividades emprendedoras dentro de la Universidad sobre ciertos montos bastante bajos para este ámbito (200 UTM). Además, las direcciones jurídicas, con respaldo de la Contraloría General de la República, han establecido un marco estricto y algo desmotivante para las actuaciones de académicos que destinan tiempo en actividades como los spin offs (Jorge Rojas, citado en Gonzalez y Jiménez, 2014, p. 137).

Por último, pareciera ser que los temas de innovación y, particularmente, de emprendimiento son percibidos por los académicos más tradicionales como nuevas tendencias que vienen a disminuir la calidad de lo exigido tanto a académicos como a estudiantes, ya que se relaciona más con el desarrollo de habilidades sociales y comunicacionales, y no puramente con la excelencia científica tecnológica.

Lo que los entrevistados observan, además, es que dicho carácter academicista del cuerpo académico de la facultad permea también la cultura estudiantil: los estudiantes buscan el trabajo solitario y dominar temas altamente específicos, así como rehúyen las actividades que impliquen un desarrollo y uso de sus habilidades sociales y comunicacionales, por considerarlas menos importantes.

\section{Nota}

El presente estudio fue realizado en el marco del proyecto "Ingeniería 2030" (código 14ENI2-26863), financiado por CORFO - Ministerio de Economía - Gobierno de Chile.

\section{Referencias bibliográficas}

Aulet, W. y Murray, F. (2013). A Tale of Two Entrepreneurs: Understanding differences in the Types of Entrepreneurship in the Economy. Recuperado de: https://papers.ssrn.com/sol3/papers.cfm?abstract_ id=2259740. DOI: $10.2139 /$ ssrn. 2259740

Bernier, L. y Hafsi, T. (2007). The changing nature of public entrepreneurship. Public Administration Review, 67(3), 488-503. DOI: $10.1111 / \mathrm{j} .1540-6210.2007 .00731 . \mathrm{x}$

Bjorklund, S. y Colbeck, C. L. (2001). The View from the Top: Leaders' Perspectives on a Decade of Change in Engineering Education.
Journal of Engineering Education, 90(1), 13-19. DOI: 10.1002/j.21689830.2001.tb00562.x

Bourque, C. (2016). La perception de l'innovation chez les chercheurs canadiens des sciences dites dures. Quaderni, (2), 81-95. DOI: 10.4000/quaderni.984

Brady, T. y Hobday, M. (2011). Projects and Innovation: Innovations and Projects. En P. Morris, J. Pinto y J. Söderlund (eds.) The Oxford Handbook of Project Management. Oxford: Oxford University Press. DOI: 10.1093/oxfordhb/9780199563142.003.0012

Brunner, J. J. (2006). Diversificación y diferenciación de la educación superior en chile en un marco internacional comparado. Recuperado desde: http://200.6.99.248/ bru487cl/files/Diversific.pdf

Clark, B. (2001). The entrepreneurial university: New foundations for collegiality, autonomy, and achievement. Higher Education Management, 13(2), 9-24. DOI: 10.1787/hemp-v13-2-en

Crawley, E., Malmqvist J., Ostlund, S. y Brodeur, D. (2007). Rethinking engineering education: The CDIO approach. Nueva York: Springer. DOI: 10.1007/978-3-319-05561-9_2

Davey, T., Plewa, C. y Struwig, M. (2011). Entrepreneurship perceptions and career intentions of international students. Education+ Training, 53(5), 335-352. DOI: 10.1108/00400911111147677

Denzin, N. y Lincoln, S. (2000). The discipline and practice of qualitative research. En N. K. Denzin y Y. S. Lincoln (Eds.), Handbook of Qualitative Research (pp. 1-28). Londres: Sage Publications. DOI: $10.1108 / 09504120610655394$

Flick, U. (2002). Introducción a la investigación cualitativa. Madrid: Ediciones Morata.

Gonzalez, H. y Jiménez, A. (2014). Inserción Laboral de Nuevos Investigadores con Grado de Doctor en Chile. Journal of Technology Management \& Innovation, 9(4), 132-148. doi: http://dx.doi.org/10.4067/ S0718-27242014000400010

Guerrero, M. y Urbano, D. (2012). The development of an entrepreneurial university. The Journal of Technology Transfer, 37(1), 43-74. DOI: $10.1007 / \mathrm{s} 10961-010-9171-\mathrm{x}$

Klein, A. y Zur, E. (2009). Entrepreneurial shareholder activism: Hedge funds and other private investors. The Journal of Finance, 64(1), 187-229. DOI: 10.2139/ssrn.913362

Krause, M. (1995). La investigación cualitativa: un campo de posibilidades y desafíos. Revista Temas de Educación, 7, 19-39.

Martinelli, A., Meyer, M. y Von Tunzelmann, N. (2008). Becoming an entrepreneurial university? A case study of knowledge exchange relationships and faculty attitudes in a medium-sized, research-oriented university. The Journal of Technology Transfer, 33(3), 259-283. DOI: 10.1007/s10961-007-9031-5 
McInnis, C. (2001). Researching the first year experience: where to from here?. Higher Education Research and Development, 20(2), 105114. DOI: $10.1080 / 07294360125188$

Mora, J. G. y Villarreal, E. (2001). Breaking down structural barriers to innovation in traditional universities. Higher Education Management, 13(2). DOI: 10.1787/hemp-v13-2-en

Morris, M., Coombes, S., Schindehutte, M. y Allen, J. (2007). Antecedents and outcomes of entrepreneurial and market orientations in a non-profit context: Theoretical and empirical insights. Journal of Leadership \& Organizational Studies, 13(4), 12-39. DOI: 10.1177/10717919070130040401

Noy, C. (2008). Sampling knowledge: The hermeneutics of snowball sampling in qualitative research. International Journal of social research methodology, 11(4), 327-344. DOI: 10.1080/13645570701401305
Oleksiyenko, A. (2002). The Entreprenuerial Response of Public Universities. CSSHE Professional file, 22, 1-17.

Souitaris, V., Zerbinati, S. y Al-Laham, A. (2007). Do entrepreneurship programmes raise entrepreneurial intention of science and engineering students? The effect of learning, inspiration and resources. Journal of Business venturing, 22(4), 566-591. DOI: doi.org/10.1016/j. jbusvent.2006.05.002

Taylor, S. y Bogdan, R. (2010). Introducción a los métodos cualitativos de investigación. Barcelona: Paidós.

World Economic Forum. (2009). Educating the next wave of entrepreneurs: Unlocking entrepreneurial capabilities to meet the global challenges of the 21st century. Switzerland: WEF. DOI: 10.2139/ ssrn.1396704 